\title{
Generalized exclusion processes: Transport coefficients
}

\author{
Chikashi Arita, ${ }^{1}$ P. L. Krapivsky, ${ }^{2}$ and Kirone Mallick ${ }^{3}$ \\ ${ }^{1}$ Theoretische Physik, Universität des Saarlandes, 66041 Saarbrücken, Germany ${ }^{*}$ \\ ${ }^{2}$ Department of Physics, Boston University, Boston, MA 02215, USA \\ ${ }^{3}$ Institut de Physique Théorique, IPhT, CEA Saclay and URA 2306, CNRS, 91191 Gif-sur-Yvette cedex, Franc‡
}

\begin{abstract}
A class of generalized exclusion processes with symmetric nearest-neighbor hopping which are parametrized by the maximal occupancy, $k \geq 1$, is investigated. For these processes on hyper-cubic lattices we compute the diffusion coefficient in all spatial dimensions. In the extreme cases of $k=1$ (symmetric simple exclusion process) and $k=\infty$ (non-interacting symmetric random walks) the diffusion coefficient is constant, while for $2 \leq k<\infty$ it depends on the density and $k$. We also study the evolution of the tagged particle, show that it exhibits a normal diffusive behavior in all dimensions, and probe numerically the coefficient of self-diffusion.
\end{abstract}

PACS numbers: 05.70.Ln, 02.50.-r, 05.40.-a

\section{INTRODUCTION}

Exclusion processes constitute an important class of lattice gases that plays a prominent role in numerous subjects including non-equilibrium statistical mechanics, soft matter, traffic models, biophysics, combinatorics and probability theory [1 9]. By definition, exclusion processes are lattice gases supplemented with stochastic hopping and obeying the constraint that at most one particle per site is allowed. In simple exclusion models, only hops to nearest-neighboring sites are allowed. These models are exactly solvable in low dimensions [4, 10, 11, and they have become benchmarks to test general theories for non-equilibrium behaviors [12 17.

Because of its ubiquity and usefulness, numerous more complicated variants of the basic exclusion process have been investigated (see [3, 9, 10] and references therein). One natural generalization is to alleviate the exclusion constraint by allowing $k$ particles per site $(k \geq 1$ is a fixed integer). More precisely, this process is defined in $d$ dimensions, say on the hyper-cubic lattice $\mathbb{Z}^{d}$, as follows: (i) each particle attempts to hop to its $2 d$ neighbors with the same unit rate to each neighbor (symmetric hopping), (ii) every hopping attempt is successful when the target site is occupied by less than $k$ particles, otherwise the hopping attempt is rejected (Fig. 1). The symmetric exclusion process (SEP) is recovered when $k=1$, whereas for $k=\infty$ the model reduces to independent random walks undergoing symmetric nearest-neighbor hopping. Letting $k$ vary from 1 to $\infty$ allows us to interpolate between a strongly interacting to a non-interacting system. (In quantum systems, restricting the maximum number of particles in a given quantum state to an integer $k$, with $1<k<\infty$, leads to the so-called Gentile statistics [18, 19 interpolating between Fermi-Dirac statistics and Bose-Einstein statistics. In this paper we consider only

\footnotetext{
*Electronic address: c.arita@physik.uni-saarland.de

${ }^{\dagger}$ Electronic address: pkrapivsky@gmail.com

¥Electronic address: kirone.mallick@cea.fr
}

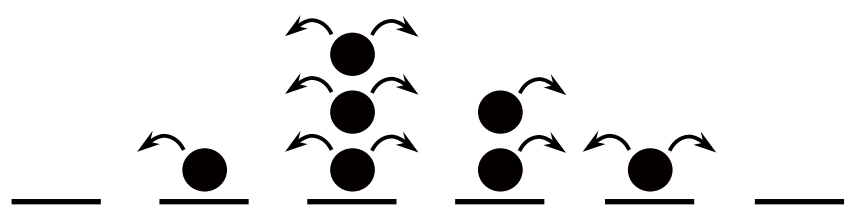

FIG. 1: GEP with $k=3$ in one dimension. Hopping into a site occupied by three particles is forbidden; other hopping events occur with the same (unit) rate. Thus the total hopping rate from every site is equal to the number of particles at the site times the number of neighboring sites which are not fully occupied.

classical lattice gases.)

Generalized exclusion processes (GEPs) with maximal occupation number $1<k<\infty$ have been studied in 20 22; see also Refs. 23 33 for other versions of GEPs. Some of these models can be mapped onto multi-species exclusion processes [34 36] but with nonconserving species. Overall, the GEPs are considerably less understood than the ordinary exclusion process - the integrability properties of the SEP do not carry over to the GEPs and a different perspective is required.

The objective of this work is to investigate the GEP at a coarse-grained level and to calculate the transport coefficients that underlie the hydrodynamic description. Section III reviews known properties of the GEP and outlines its macroscopic (i.e., hydrodynamic) regime which is governed by a diffusion equation. We also formulate our main result, namely a parametric representation of the diffusion coefficient. In Sec. III] we present the derivation of the diffusion coefficient. In Sec. IV] stationary density profiles are computed and compared with simulations. In Sec. V], we examine the evolution of a tagged particle in the GEP in various spatial dimensions. We derive the self-diffusion coefficient using a mean-field approximation. We also probe the self-diffusion coefficient numerically and show a reasonable qualitative agreement with mean-field predictions. We summarize our results, and mention remaining challenges and possible extensions, in Sec. VI. 


\section{HYDRODYNAMIC BEHAVIOR}

For the generalized exclusion process with symmetric hopping, steady states are remarkably simple and are given by a product measure [2, 5, 6, 27]. In other words, one only needs to know the probabilities $P_{j}$ to have $j$ particles per site and the stationary weight of any configuration factors into the product of these basic probabilities. The basic probabilities are given by an elementary formula [6, 25, 27]

$$
P_{j}=\frac{\lambda^{j}}{j !} \frac{1}{Z_{k}(\lambda)} .
$$

To justify (1) it suffices to use the factorization and verify that the flow $(i, j) \Longrightarrow(i-1, j+1)$, which is given by $i P_{i} P_{j}$, is equal to the flow $(i-1, j+1) \Longrightarrow(i, j)$, which is given by $(j+1) P_{i-1} P_{j+1}$. With the choice (1), we indeed get $i P_{i} P_{j}=(j+1) P_{i-1} P_{j+1}$. The "partition function' $Z_{k}(\lambda)$, which appears in Eq. (1), is fixed by the normalization requirement $\sum_{0 \leq j \leq k} P_{j}=1$. It is equal to an incomplete exponential function:

$$
Z_{k}(\lambda)=\sum_{j=0}^{k} \frac{\lambda^{j}}{j !}
$$

The 'fugacity' parameter $\lambda$ is implicitly determined by the density $\rho$ :

$$
\rho=\sum_{j=0}^{k} j P_{j}=\lambda \frac{Z_{k-1}(\lambda)}{Z_{k}(\lambda)} .
$$

Furthermore, GEPs with rather general hopping rates depending on the number of particles on the exit site have been also studied; see, e.g. [6, 20, 24, 25, 27, 37. In all these models the steady-state probabilities are also given by a product measure. We emphasize that this product measure structure is akin to that of the zero-range process [38, 39] although the two processes are fundamentally different (in the GEP, the jump rate of a particle does depend on the state of the target site, contrary to what is assumed in the zero-range process).

In order to study the dynamics of the system, the knowledge of the steady-state distribution is not sufficient and a full description of the evolution requires the complete spectrum and eigenstates of the evolution matrix. Yet the large scale "hydrodynamic" behavior is conceptually simple. The only relevant hydrodynamic variable is density and it evolves according to a diffusion equation. In one dimension, for instance, it reads

$$
\frac{\partial \rho}{\partial t}=\frac{\partial}{\partial x}\left[D_{k}(\rho) \frac{\partial \rho}{\partial x}\right] .
$$

This generic result is valid for lattice gases with symmetric hopping [2, 5, 6]. Thus the detailed microscopic rules underlying the dynamics of the lattice gas play little role; namely, they are all encapsulated in a single density-dependent function, the diffusion coefficient.
The determination of the diffusion coefficient is in principle a very difficult problem as we do not assume the lattice gas to be dilute. For the GEP, the diffusion coefficient $D_{k}$ is known in the extreme cases, namely for symmetric random walks $(k=\infty)$ and for the $\operatorname{SEP}(k=1)$. In both these cases the diffusion coefficient is constant; with our choice of the hopping rates, we have

$$
D_{1}=D_{\infty}=1
$$

For other maximal occupancies $(1<k<\infty)$, the diffusion coefficient is density-dependent. This already follows from the asymptotic behaviors

$$
D_{k}(\rho)= \begin{cases}1 & \rho \rightarrow 0 \\ k & \rho \rightarrow k .\end{cases}
$$

The small-density asymptotic corresponds to the diffusion of a single particle in the empty system, while the behavior in the $\rho \rightarrow k$ limit can be understood by considering a single vacancy in the fully occupied system.

The computation of $D_{k}(\rho)$ for all $k$ will be presented in section III. We will show that

$$
D_{k}=\Lambda_{k}-\rho \frac{d \Lambda_{k}}{d \rho}, \quad \Lambda_{k}(\rho)=1-P_{k}(\rho) .
$$

Using (1) and (3) one obtains the parametric representation of $\Lambda_{k}(\rho)$ :

$$
\rho=\lambda \Lambda_{k}(\lambda), \quad \Lambda_{k}(\lambda)=\frac{Z_{k-1}(\lambda)}{Z_{k}(\lambda)} .
$$

These formulas apply to all $1 \leq k \leq \infty$ including the extreme cases. For the SEP we have $\Lambda_{1}=1-\rho$, while for random walks $\Lambda_{\infty}=1$; in both cases we recover (5). In all other cases $(2 \leq k<\infty)$, the diffusion coefficients $D_{k}(\rho)$ are monotonically increasing convex functions of the density (as illustrated in Fig. 2 for $k=2,3,4,5$ ).

For lattice gases in higher dimensions, the density generally satisfies a diffusion equation

$$
\frac{\partial \rho}{\partial t}=\sum_{a, b=1}^{d} \frac{\partial}{\partial x_{a}}\left[D^{a b}(\rho) \frac{\partial \rho}{\partial x_{b}}\right]
$$

with a $d \times d$ diffusion matrix $D^{a b}(\rho)$. An ordinary diffusion process (e.g., a symmetric random walk) is macroscopically isotropic, so the diffusion matrix is scalar: $D^{a b}(\rho)=\delta^{a b} D(\rho)$. Generally the diffusion matrix is symmetric, $D^{a b}(\rho)=D^{b a}(\rho)$, and for lattice gases on $\mathbb{Z}^{d}$ the symmetry of the lattice limits the number of independent matrix elements to two: All diagonal elements are equal [we denote them by $D(\rho)$ ], and all off-diagonal are also equal [we denote them by $\widehat{D}(\rho)$ ]. In three dimensions, for instance, the diffusion matrix is

$$
\mathbf{D}(\rho)=\left[\begin{array}{lll}
D(\rho) & \widehat{D}(\rho) & \widehat{D}(\rho) \\
\widehat{D}(\rho) & D(\rho) & \widehat{D}(\rho) \\
\widehat{D}(\rho) & \widehat{D}(\rho) & D(\rho)
\end{array}\right] .
$$




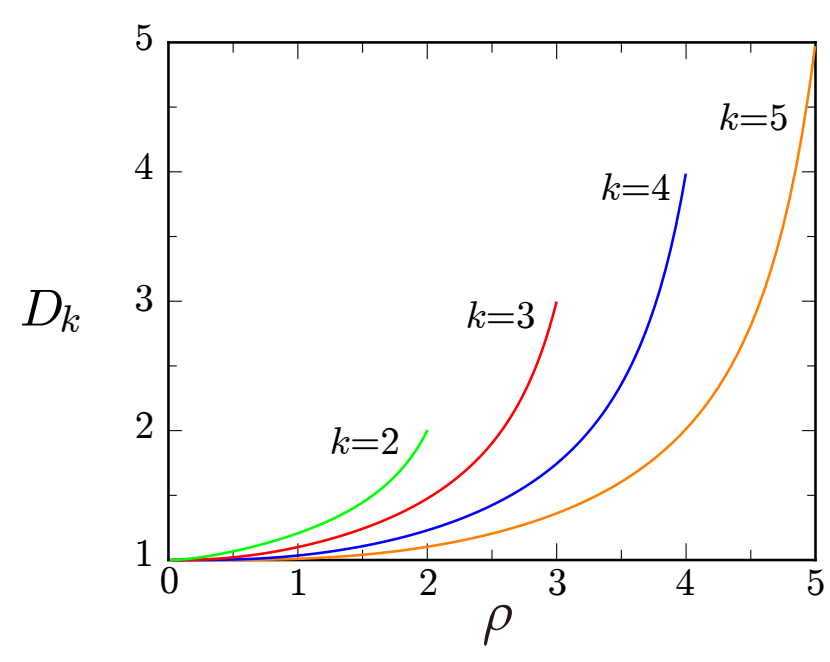

FIG. 2: Diffusion coefficient (7) as a function of density for the GEP with $k=2,3,4,5$.

For lattice gases on $\mathbb{Z}^{d}$ in which each particle occupies a single lattice site one expects the diffusion matrix to be scalar and the diffusion coefficient to be strictly positive [40]. As far as we know these physically obvious assertions have not been proved in full generality. In the next section, we calculate $D_{k}(\rho)$ for all $k$. Our derivation implies that for the GEP the diffusion process is indeed macroscopically isotropic, and our explicit results show that the diffusion coefficients satisfy $D_{k}(\rho)>1$ and they are independent of the spatial dimension.

\section{DIFFUSION COEFFICIENT}

In this section, we calculate the diffusion coefficient for the GEP that appears in Eq. (9). As a warm-up, we recall the well-known case of the SEP. Then we show the crucial difference between the GEPs with $2 \leq k<\infty$ and the SEP, namely the presence of the higher-order correlators, which makes impossible an elementary derivation of the diffusion coefficient. Fortunately, the understanding of the equilibrium in the GEP and a perturbative expansion around the equilibrium gives a method for deriving the diffusion coefficient for the GEPs with $2 \leq k<\infty$. We present a detailed derivation of $D_{2}(\rho)$ in one and higher dimensions. The case of arbitrary $k$ is outlined at the end of this section.

A configuration of the SEP on a one-dimensional lattice is fully described by binary variables $n_{j}(t)$ : If the site $j \in \mathbb{Z}$ is empty, $n_{j}(t)=0$; if it is occupied, $n_{j}(t)=1$. In an infinitesimal time interval $d t$, the particle hops from site $j$ to site $j+1$ with probability $n_{j}\left(1-n_{j+1}\right) d t$. This choice assures that the hopping event happens only when the site $j$ is occupied and the site $j+1$ is empty. Taking into account all possible hops one finds that the average density evolves according to

$$
\begin{aligned}
\frac{d\left\langle n_{j}\right\rangle}{d t} & =\left\langle n_{j-1}\left(1-n_{j}\right)+n_{j+1}\left(1-n_{j}\right)\right\rangle \\
& -\left\langle n_{j}\left(1-n_{j-1}\right)+n_{j}\left(1-n_{j+1}\right)\right\rangle
\end{aligned}
$$

which simplifies to the discrete diffusion equation

$$
\frac{d\left\langle n_{j}\right\rangle}{d t}=\left\langle n_{j-1}\right\rangle-2\left\langle n_{j}\right\rangle+\left\langle n_{j+1}\right\rangle
$$

The remarkable cancellation of the higher-order correlation functions allows one to prove the validity of the hydrodynamic limit without further assumptions - no need to use the absence of correlations in the steady state. By definition, in the hydrodynamic limit the average density varies on the scales greatly exceeding the lattice spacing. Therefore we write $\left\langle n_{j}(t)\right\rangle=\rho(x, t)$; the notation $x=j$ emphasizes that we are switching to the continuum description. We then expand $\left\langle n_{j \pm 1}\right\rangle$ in Taylor series

$$
\left\langle n_{j \pm 1}\right\rangle=\rho \pm \rho_{x}+\frac{1}{2} \rho_{x x}+\cdots
$$

and recast the set of difference-differential equations 12 into a classical diffusion equation, namely Eq. (4) with $D_{1}=1$. In higher dimensions, the cancellation still holds; in two dimensions, for instance,

$$
\begin{aligned}
\frac{d\left\langle n_{i, j}\right\rangle}{d t} & =\left\langle n_{i, j-1}\right\rangle+\left\langle n_{i, j+1}\right\rangle+\left\langle n_{i-1, j}\right\rangle+\left\langle n_{i+1, j}\right\rangle \\
& -4\left\langle n_{i, j}\right\rangle .
\end{aligned}
$$

Therefore the hydrodynamic description is again the classical diffusion equation $\rho_{t}=\rho_{x x}+\rho_{y y}$.

Consider now the simplest GEP different from the SEP, namely the GEP with $k=2$ on a one-dimensional lattice. The occupation number $n_{j}$ is either 0 , or 1 , or 2 when $k=2$. The process $\left(n_{j}, n_{j+1}\right) \Longrightarrow\left(n_{j}-1, n_{j+1}+1\right)$ proceeds with rate

$$
n_{j} F\left(n_{j+1}\right), \quad F(n)=1-\frac{n(n-1)}{2} .
$$

Therefore the average density evolves according to

$$
\begin{aligned}
\frac{d\left\langle n_{j}\right\rangle}{d t} & =\left\langle\left[n_{j-1}+n_{j+1}\right] F\left(n_{j}\right)\right\rangle \\
& -\left\langle n_{j}\left[F\left(n_{j-1}\right)+F\left(n_{j+1}\right)\right]\right\rangle .
\end{aligned}
$$

In contrast with the case of the SEP, higher-order correlation functions do not cancel as it is obvious from an explicit representation of the right-hand side of 16$)$ :

$$
\begin{aligned}
\frac{d\left\langle n_{j}\right\rangle}{d t} & =\left\langle n_{j-1}\right\rangle-2\left\langle n_{j}\right\rangle+\left\langle n_{j+1}\right\rangle \\
& +\frac{1}{2}\left\langle n_{j}\left[n_{j-1}^{2}+n_{j+1}^{2}\right]-\left[n_{j-1}+n_{j+1}\right] n_{j}^{2}\right\rangle .
\end{aligned}
$$

It is often possible to advance for lattice gases of the gradient type [2, 6]. These are lattice gas models in which the current $J_{j, j+1}$ of particles moving from any site $j$ to $j+1$ can be written as a discrete gradient. For instance, 
the SEP is the gradient lattice gas since $J_{j, j+1}=n_{j}-$ $n_{j+1}$. For the GEP with $k=2$, the current

$$
J_{j, j+1}=n_{j}-n_{j+1}+\frac{1}{2}\left[n_{j+1} n_{j}^{2}-n_{j} n_{j+1}^{2}\right]
$$

is obviously not a discrete gradient. Generally all GEPs with $2 \leq k<\infty$ are non-gradient lattice gases.

We now outline the idea of a perturbative approach which we shall use to establish the diffusion coefficient in non-gradient lattice gases, and then return to the GEP.

\section{A. Perturbative Approach}

For non-gradient lattice gases it is sometimes possible to study the hydrodynamic regime in the realm of a perturbative approach. The idea is to ignore correlations. This is true in the equilibrium. In the evolving state, the presence of local density gradients induces long-ranged correlations, but in numerous lattice gases these correlations vanish to first order in the density difference. This has been rigorously established (in all spatial dimensions) for lattice gases with hard-core exclusion [41, and it is expected to apply to a much larger class of models. There can be appreciable correlations in the earlier time regime, but we are interested in the hydrodynamic limit which, by definition, describes the evolution close to equilibrium. The vanishing of correlations in the first order in the density gradient implies that in the hydrodynamic regime our perturbative treatment leads to exact predictions for the diffusion coefficient.

To appreciate the validity of a perturbative approach it is useful to compare the situation with kinetic theory [2, 7, 42]. Recall that the Boltzmann equation, even though it is mean-field in nature (as it is based on the assumption of molecular chaos), is asymptotically exact in the hydrodynamic regime, so the emerging transport coefficients are exact. In the context of kinetic theory the main challenge is technical - even for dilute monoatomic gases (e.g., for hard spheres gas), it has not been possible to extract transport coefficients analytically 42. Lattice gases are much more tractable, so even for dense lattice gases the computing of the diffusion coefficient is occasionally feasible. The crucial ingredient is the understanding of the equilibrium state. We emphasize that for lattice gases of gradient type (e.g., for the KatzLebowitz-Spohn model with symmetric hopping [1, 43. and for repulsion processes 44]) when computations using a Green-Kubo formula become feasible, the results for the diffusion coefficient agree with predictions derived using the perturbative approach. Further, for lattice gases of non-gradient type whenever it was possible to apply the perturbative approach (see [45, 46]), the predictions for the diffusion coefficient were again exact as it was evidenced through rigorous analyses, mappings to gradient type lattice gases, and comparisons with simulations.

\section{B. GEP with $k=2$}

To implement the perturbative approach for the GEP with $k=2$ on the one-dimensional lattice, we first replace (16) by

$$
\begin{aligned}
\frac{d\left\langle n_{j}\right\rangle}{d t} & =\left[\left\langle n_{j-1}\right\rangle+\left\langle n_{j+1}\right\rangle\right]\left\langle F\left(n_{j}\right)\right\rangle \\
& -\left\langle n_{j}\right\rangle\left[\left\langle F\left(n_{j-1}\right)\right\rangle+\left\langle F\left(n_{j+1}\right)\right\rangle\right] .
\end{aligned}
$$

In the hydrodynamic limit we write $\left\langle n_{j}(t)\right\rangle=\rho(x, t)$ and we use Eq. 13 for $\left\langle n_{j \pm 1}(t)\right\rangle$ to yield

$$
\left\langle n_{j-1}\right\rangle+\left\langle n_{j+1}\right\rangle=2 \rho+\rho_{x x} .
$$

Hereinafter, we keep the terms which survive in the hydrodynamic limit, e.g., in Eq. 20 we have dropped $\frac{1}{12} \rho_{x x x x}$ and the following terms with higher derivatives.

The average $\langle F(n)\rangle$ has a neat form

$$
\langle F(n)\rangle=1-P_{2}(\rho),
$$

which is obvious from the definition of the process (the hopping can occur only when the target site hosts less than two particles). We shall use the shorthand notation $1-P_{2}(\rho)=\Lambda_{2}(\rho)$.

In the hydrodynamic limit $\left\langle F\left(n_{j-1}\right)\right\rangle+\left\langle F\left(n_{j+1}\right)\right\rangle$ turns into $\Lambda_{2}[\rho(x-1)]+\Lambda_{2}[\rho(x+1)]$, which is expanded to yield

$$
2 \Lambda_{2}(\rho)+\Lambda_{2}^{\prime}(\rho) \rho_{x x}+\Lambda_{2}^{\prime \prime}(\rho) \rho_{x}^{2} .
$$

Inserting all these expansions into (19) we arrive at

$$
\rho_{t}=\left[\Lambda_{2}(\rho)-\rho \Lambda_{2}^{\prime}(\rho)\right] \rho_{x x}-\rho \Lambda_{2}^{\prime \prime}(\rho) \rho_{x}^{2} .
$$

This equation can be re-written as the diffusion equation (4) with diffusion coefficient

$$
D_{2}=\Lambda_{2}(\rho)-\rho \Lambda_{2}^{\prime}(\rho) .
$$

Recall that, for $k=2$, we have

$$
\rho=\frac{\lambda+\lambda^{2}}{1+\lambda+\frac{1}{2} \lambda^{2}}, \quad \Lambda_{2}=\frac{1+\lambda}{1+\lambda+\frac{1}{2} \lambda^{2}}
$$

from which we find an explicit expression for $\Lambda_{2}(\rho)$ :

$$
\Lambda_{2}(\rho)=\frac{1-\rho+\sqrt{1+2 \rho-\rho^{2}}}{2} .
$$

Inserting this into (24) yields an explicit expression of the diffusion coefficient

$$
D_{2}(\rho)=\frac{1+\rho+\sqrt{1+2 \rho-\rho^{2}}}{2 \sqrt{1+2 \rho-\rho^{2}}} .
$$

We now consider the GEP with $k=2$ in arbitrary dimension. In two dimensions, for instance, the average density satisfies

$$
\begin{aligned}
& \frac{d\left\langle n_{i, j}\right\rangle}{d t}=\left\langle\left(n_{i-1, j}+n_{i+1, j}+n_{i, j-1}+n_{i, j+1}\right) F\left(n_{i, j}\right)\right\rangle \\
& -\left\langle n_{i, j}\left[F\left(n_{i-1, j}\right)+F\left(n_{i+1, j}\right)+F\left(n_{i, j-1}\right)+F\left(n_{i, j+1}\right)\right]\right\rangle
\end{aligned}
$$


which in the hydrodynamic limit becomes

$$
\rho_{t}=\partial_{x}\left(D_{2} \rho_{x}\right)+\partial_{y}\left(D_{2} \rho_{y}\right)
$$

with $D_{2}$ given by Eq. 24 as in one dimension. The same holds in any spatial dimension, namely the GEP is described by the diffusion equation

$$
\rho_{t}=\nabla \cdot\left(D_{2} \nabla \rho\right)
$$

where the diffusion coefficient is given by a universal formula (24) valid in arbitrary dimension. The symmetric GEP is therefore isotropic on the hydrodynamic scale; namely, it is described by the scalar diffusion coefficient.

\section{GEP with arbitrary $k$}

For the GEP with arbitrary $k$ the analysis is similar to the one presented above. The process $\left(n_{j}, n_{j+1}\right) \Longrightarrow$ $\left(n_{j}-1, n_{j+1}+1\right)$ proceeds with rate 15$)$, where we only need to modify $F(n)$ to

$$
F(n)=1-\frac{n(n-1) \cdots(n-k+1)}{k !} .
$$

It suffices to consider the one-dimensional case as the results for the diffusion coefficient are independent of the spatial dimensionality. Equations $(16)$ and $\sqrt{19}$, with $F(n)$ given by $(30$, remain valid. Equations (20)-24 also hold if we replace $\Lambda_{2}$ by $\Lambda_{k}$, the probability that a site is not fully occupied; for instance, Eq. 21 becomes $\langle F(n)\rangle=1-P_{k}(\rho) \equiv \Lambda_{k}(\rho)$. Thus the diffusion coefficient is indeed given by the announced expression 7). For $k \geq 5$ an explicit expression for $\Lambda_{k}(\rho)$ is apparently impossible to deduce, but we can use a parametric expression (8) which follows from (1), (3), and the definition $\Lambda_{k}(\rho)=1-P_{k}(\rho)$.

\section{STATIONARY DENSITY PROFILES}

In the previous section we calculated the diffusion coefficient for the GEP using a perturbative approach. In this section, we present a non-direct test of our predictions. Specifically, we shall calculate stationary density profiles in one and two dimensions and compare these theoretical predictions with simulation results. We will show that the diffusion equation with the diffusion coefficient given by Eqs. (7) and (8) provides an accurate description of the system at a macroscopic scale.

\section{A. One-dimensional density profiles}

Consider the GEP on the interval $(0, L)$ with boundary conditions

$$
\rho(0)=\rho_{0}, \quad \rho(L)=\rho_{1}
$$

Solving $D_{k}(\rho) \frac{d \rho}{d x}=$ const, subject to 31 , we obtain

$$
\frac{\int_{\rho_{0}}^{\rho} d r D_{k}(r)}{\int_{\rho_{0}}^{\rho_{1}} d r D_{k}(r)}=\frac{x}{L}
$$

Let us focus on a special case when the right boundary is a sink: $\rho_{1}=0$. To simplify formulas we write $\rho_{0}=n$. When $k=2$, the integrals on the left-hand side of 32 can be explicitly determined to yield an implicit representation of the stationary density profile $\rho(x)$ :

$$
\frac{1+\frac{\pi}{2}+\rho-\sqrt{1+2 \rho-\rho^{2}}+2 \arcsin \left(\frac{\rho-1}{\sqrt{2}}\right)}{1+\frac{\pi}{2}+n-\sqrt{1+2 n-n^{2}}+2 \arcsin \left(\frac{n-1}{\sqrt{2}}\right)}=1-\frac{x}{L} .
$$

In the case of the maximal density on the left boundary, $\rho_{0}=n=2$, we get

$$
\frac{1}{2}-\frac{\rho-\sqrt{1+2 \rho-\rho^{2}}+2 \arcsin \left(\frac{\rho-1}{\sqrt{2}}\right)}{\pi+2}=\frac{x}{L},
$$

see Fig. 3 .

In the general case of arbitrary $k$ we use (7) and (8) and establish the following parametric representation

$$
\frac{\int_{0}^{\lambda} d \mu\left[\Lambda_{k}(\mu)\right]^{2}}{\int_{0}^{\ell} d \mu\left[\Lambda_{k}(\mu)\right]^{2}}=1-\frac{x}{L}, \quad n=\ell \Lambda_{k}(\ell) .
$$

The maximal density on the left boundary, $\rho_{0}=n=k$, corresponds to $\ell=\infty$. The density profiles 34 in this situation,

$$
\frac{\int_{0}^{\lambda} d \mu\left[\Lambda_{k}(\mu)\right]^{2}}{\int_{0}^{\infty} d \mu\left[\Lambda_{k}(\mu)\right]^{2}}=1-\frac{x}{L}, \quad \rho=\lambda \Lambda_{k}(\lambda)
$$

are plotted in Fig. 3 .

\section{B. The GEP in an annulus}

For the GEP in the annulus $a \leq R \leq L$, we solve $R D_{k}(\rho) \frac{d \rho}{d R}=$ const, subject to the boundary conditions $\rho(a)=\rho_{0}$ and $\rho(L)=\rho_{1}$, and get

$$
\frac{\int_{\rho_{0}}^{\rho} d r D_{k}(r)}{\int_{\rho_{0}}^{\rho_{1}} d r D_{k}(r)}=\frac{\ln (R / a)}{\ln (L / a)}
$$

Let us look more carefully at the case of $k=2$ with boundary densities $\rho_{1}=0, \rho_{0}=2$ (the density on the inner circle is maximal). We use dimensionless variables $\alpha=a / L$ and $\xi=R / L$, so that $0<\alpha \leq \xi \leq 1$. With these choices, Eq. 36 becomes

$$
\frac{1}{2}+\frac{\rho-\sqrt{1+2 \rho-\rho^{2}}+2 \arcsin \left(\frac{\rho-1}{\sqrt{2}}\right)}{\pi+2}=\frac{\ln \xi}{\ln \alpha} .
$$

This density profile is compared with simulation results on Fig. 4 . 


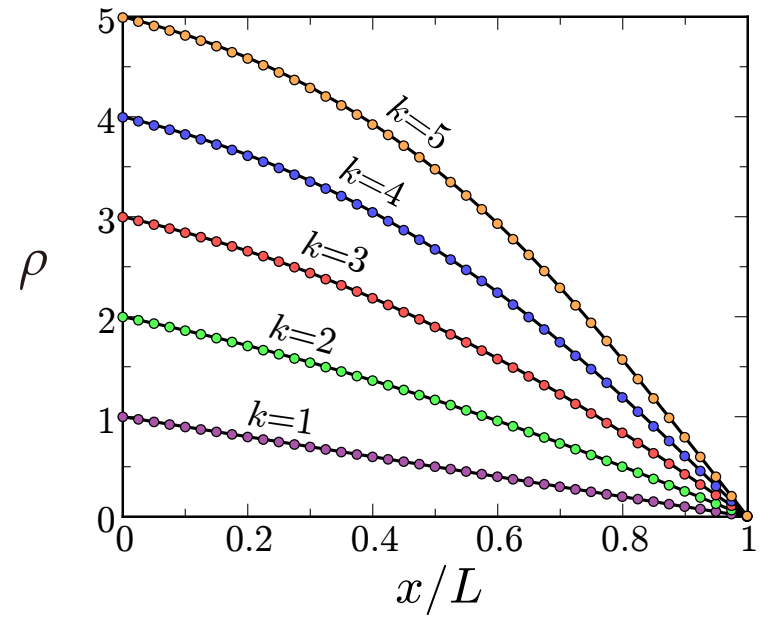

FIG. 3: Stationary density profiles versus $x / L$ for the GEP with $k=1,2,3,4,5$ on a segment with $L=10^{3}$. The solid lines are theoretical predictions in the case of extremal boundary densities, $\rho_{0}=k$ and $\rho_{1}=0$. For the $\operatorname{SEP}(k=1)$, the density profile is linear; for $k=2$, the density profile is given by (33), and generally it is extracted from Eq. (35). Simulation results (shown by $\bullet$ ) were obtained by averaging over the time window $5 \times 10^{6} \leq t \leq 10^{7}$.

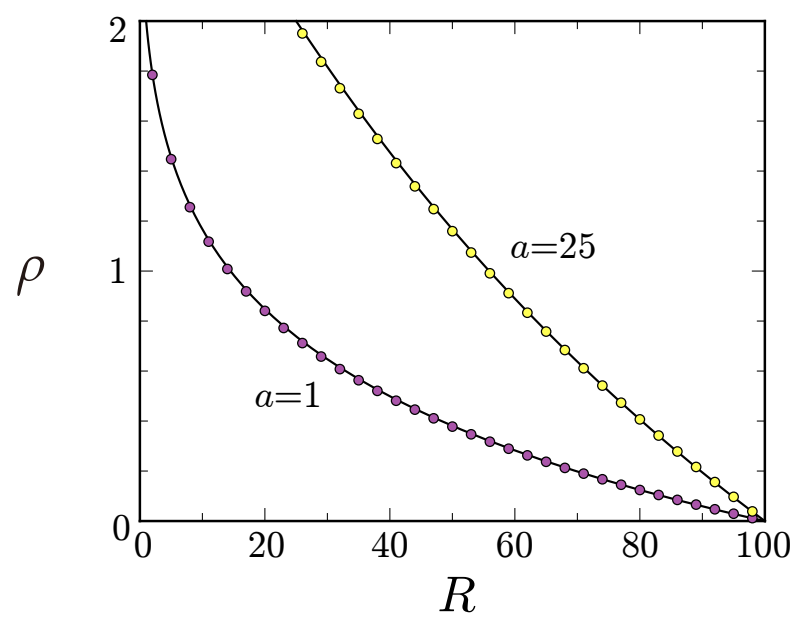

FIG. 4: Stationary density profiles versus $R$ for the GEP with $k=2$ in the annulus with external radius $L=100$. Dots are simulation results which were obtained by averaging over $5 \times 10^{6} \leq t \leq 10^{7}$. Solid lines are theoretical predictions given by (37) with $\alpha=1 / 4$ in the case of $a=25$ and by (38) when $a=1$.

For the GEP in the annulus $a \leq R \leq L$, the usage of the continuum (diffusion equation) approach is somewhat questionable near the inner circle if $a=O(1)$. Indeed, we cannot even talk about a circle on a lattice if its radius is comparable with the lattice spacing. Nevertheless, let us use Eq. (36), again with $k=2$ and $\left(\rho_{0}, \rho_{1}\right)=(2,0)$, in the extreme case of $a=1$. Equation (36) becomes

$$
\frac{1}{2}-\frac{\rho-\sqrt{1+2 \rho-\rho^{2}}+2 \arcsin \left(\frac{\rho-1}{\sqrt{2}}\right)}{\pi+2}=\frac{\ln R}{\ln L} .
$$

Choosing the inner radius equal to lattice spacing is essentially equivalent to the simplest lattice setting with reservoir connected to the origin and postulating that whenever a particle leaves the origin, a particle from reservoir is immediately added, so the density at the origin remains maximal $\rho_{0}=2$. There is also a sink at the circle $R=L$; that is, whenever a particle at a site on distance $<L$ hops and gets outside this circle, it leaves the system forever. On distances $R \gg 1$ the profile (38) should become asymptotically exact. Figure 4 shows an excellent agreement between theory and simulations over the entire range $1 \leq R \leq L$.

To emphasize the difference between one and two dimensions let us consider the GEP with $k=2$ and boundary densities $\left(\rho_{0}, \rho_{1}\right)=(2,0)$ and compare the density profiles $(33)$ and $(38)$. In one dimension, the intermediate density $\rho_{*}=\left(\rho_{0}+\rho_{1}\right) / 2$, i.e., $\rho_{*}=1$ in our case, is reached at

$$
\frac{x_{*}}{L}=\frac{1}{2}+\frac{\sqrt{2}-1}{\pi+2}=0.580561 \ldots,
$$

while in two dimensions this happens at

$$
\frac{\ln R_{*}}{\ln L}=\frac{1}{2}+\frac{\sqrt{2}-1}{\pi+2},
$$

which is much closer to the source, $R_{*} \sim L^{0.580561}$.

Second, we compare the total (average) number of particles. In one dimension we integrate by part to get

$$
N=\int_{0}^{L} d x \rho(x)=\int_{0}^{2} d \rho x(\rho) .
$$

Using (33) we perform the integration and find

$$
N=\frac{3 \pi+2}{2 \pi+4} L
$$

In two dimensions, we similarly find

$$
N=\int_{0}^{L} d R 2 \pi R \rho(R)=\pi \int_{0}^{2} d \rho R^{2}(\rho) .
$$

The dominant part of the integral in 43 is gathered near $\rho=0$. Expanding the left-hand side of 38 we find

$$
\frac{\ln R}{\ln L}=1-\frac{2}{\pi+2} \rho-\frac{1}{3(\pi+2)} \rho^{3}+\frac{1}{2(\pi+2)} \rho^{4}+\cdots .
$$

Equation 43 becomes

$$
\frac{N}{\pi L^{2}} \simeq \int_{0}^{\infty} d \rho \exp \left[-\ln L \frac{4 \rho+\frac{2}{3} \rho^{3}-\rho^{4}}{\pi+2}\right]
$$


which gives

$$
N=\frac{\pi(\pi+2)}{4} \frac{L^{2}}{\ln L}\left[1+\frac{C_{2}}{(\ln L)^{2}}+\frac{C_{3}}{(\ln L)^{3}}+\cdots\right]
$$

with $C_{2}=-\left(\frac{\pi+2}{4}\right)^{3}, C_{3}=6\left(\frac{\pi+2}{4}\right)^{4}$, etc. Thus the convergence to the leading asymptotic behavior is slow in two dimensions.

For arbitrary $k$, let us choose again $\rho_{1}=0$ and $\rho_{0}=k$. the density profile is implicitly given by

$$
\frac{\int_{0}^{\lambda} d \mu\left[\Lambda_{k}(\mu)\right]^{2}}{\int_{0}^{\infty} d \mu\left[\Lambda_{k}(\mu)\right]^{2}}=1-\frac{\ln R}{\ln L} .
$$

In the small $\rho$ limit, we get

$$
\frac{\ln R}{\ln L}=1-\frac{\rho}{I_{k}}+\cdots, \quad I_{k}=\int_{0}^{\infty} d \mu\left[\Lambda_{k}(\mu)\right]^{2},
$$

and the leading asymptotic behavior of the total average number of particles is

$$
N \simeq \frac{\pi I_{k}}{2} \frac{L^{2}}{\ln L}
$$

where the coefficients $I_{k}$ can be evaluated numerically (e.g. $I_{3}=4.29139 \ldots$...

\section{SELF-DIFFUSION COEFFICIENT}

Even an equilibrium situation (in which the density is spatially uniform) possesses interesting non-equilibrium features. One important example is the phenomenon of self-diffusion. In this section we investigate the evolution of a tagged particle in the GEP at equilibrium. We assume that the tagged particle is identical to the host particles, so it merely carries a tag. Asymptotically, the tagged particle exhibits a diffusive behavior, so it suffices to compute the coefficient of self-diffusion. This problem is easy to pose, but there has been little progress even for simplest lattice gases. For instance, the coefficient of self-diffusion is unknown for the SEP in two and higher dimensions, it is only known 47] that the coefficient of self-diffusion is a smooth function of the density.

Consider first the one-dimensional case. We tag a particle which is initially at $x(0)=0$ (without loss of generality) and we look at its position $x(t)$ in the long time limit. Generically, we expect a diffusive behavior. Thus the first two averages are $\langle x\rangle=0$ and $\left\langle x^{2}\right\rangle \sim t$, and it suffices to determine the self-diffusion coefficient

$$
\lim _{t \rightarrow \infty} \frac{\left\langle x^{2}\right\rangle}{2 t}=\mathcal{D}_{k}(\rho) .
$$

The self-diffusion coefficient $\mathcal{D}_{k}$ generally differs from the diffusion coefficient $D_{k}$. We have $\mathcal{D}_{\infty}=D_{\infty}=1$ for non-interacting random walks. For $k<\infty$, the inequality $\mathcal{D}_{k}<D_{k}$ is physically apparent, although it may be difficult to prove.

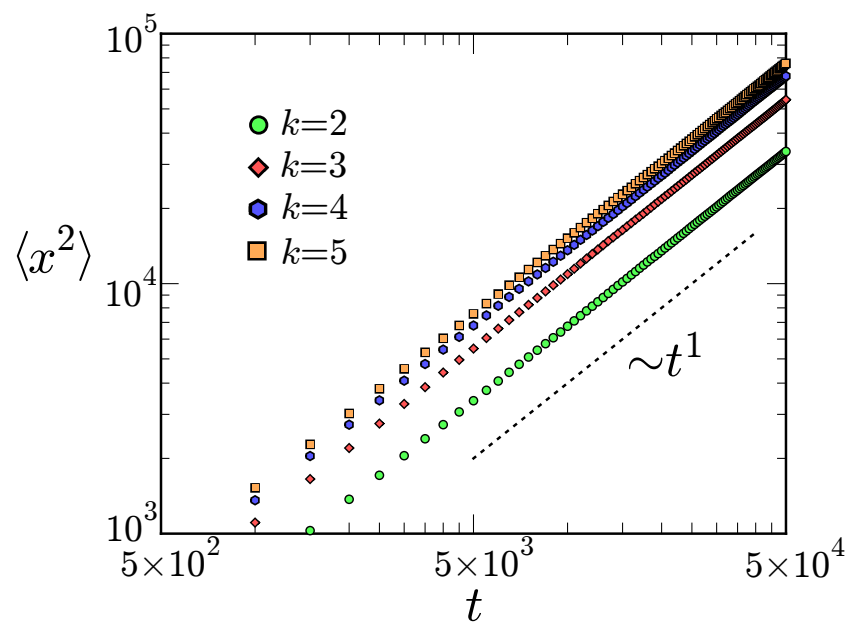

FIG. 5: Mean-square displacement of the tagged particle vs time for the GEP in one dimension. The maximal occupancy varies between $k=2$ and $k=5$. Simulations were performed on the ring of length $10^{3}$.

For the SEP in one dimension, the self-diffusion coefficient vanishes: $\mathcal{D}_{1}=0$. Indeed, the ordering between the particles is conserved and this leads to anomalously slow sub-diffusive behavior [48 52]: $\left\langle x^{2}\right\rangle_{\mathrm{SEP}, d=1} \sim t^{1 / 2}$. This is an exceptional feature; the normal diffusion is recovered for the SEP in dimensions higher than 1. For the GEP with $k \geq 2$, the phenomenon of self-diffusion is not pathological even in one dimension, viz. the selfdiffusion coefficient $\mathcal{D}_{k}(\rho)$ is positive. Moreover, $\mathcal{D}_{k}(\rho)$ is a monotonically decreasing function of $\rho$ in the interval $0<\rho<k$ with asymptotic behaviors

$$
\mathcal{D}_{k}(\rho)= \begin{cases}1 & \rho \rightarrow 0, \\ 0 & \rho \rightarrow k\end{cases}
$$

For lattice gases in $d>1$ dimensions, the spread of the tagged particle is generically described by a matrix. For the GEP on the hyper-cubic lattice, and generally for lattice gases on $\mathbb{Z}^{d}$ where each particle occupies only one site, one expects the self-diffusion process to be isotropic on the hydrodynamic scales. This has been proved only for the SEP, see [53, so it remains conjectural for the GEPs with $2 \leq k<\infty$.

There is one important feature which distinguishes the self-diffusion coefficient from the diffusion coefficient, viz. the self-diffusion coefficient certainly depends on the dimensionality: $\mathcal{D}_{k}(\rho, d)$. The extreme density behaviors of the self-diffusion coefficient $\mathcal{D}_{k}(\rho, d)$ with $k \geq 2$ are universal and given by (51) in all dimensions.

We performed simulations to probe the self-diffusion coefficient on lattices with $L^{d}$ sites, in $d=1,2,3$ dimensions, with periodic boundaries. The sizes of the simulated systems are $L=10^{3}, 32,10$ for $d=1,2,3$, respectively. The number of simulation runs for each set of parameters $(k, d, \rho)$ is $2 \times 10^{5} /\left(L^{d} \rho\right)$, and we tagged all the $L^{d} \rho$ particles in each run. Thus $\langle\cdot\rangle$ is the aver- 


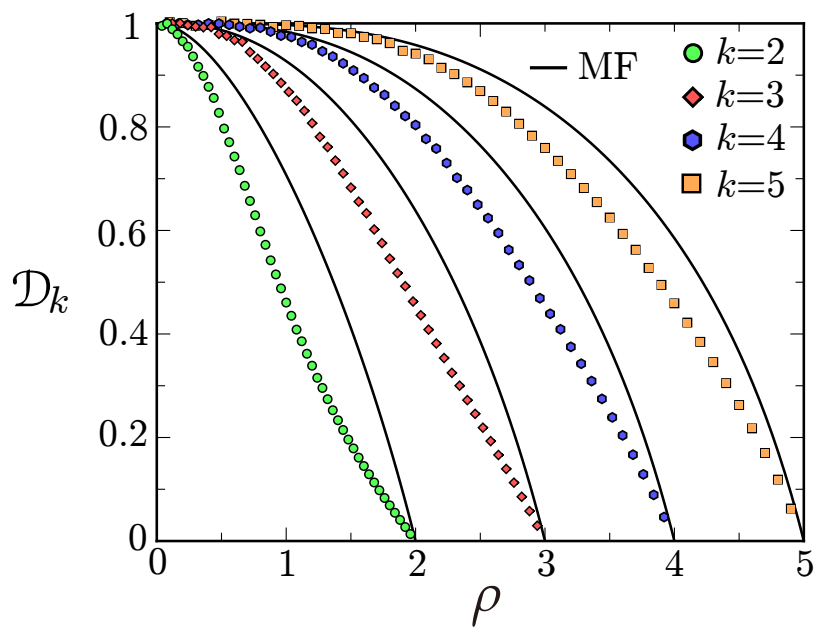

FIG. 6: Coefficient of self-diffusion vs density for the GEP in one dimension; the maximal occupancy varies between $k=2$ and $k=5$. Dots represent simulation results for the GEP in one dimension. Solid lines are the mean-field predictions, Eq. (52).

age over effectively $2 \times 10^{5}$ tagged particles. We checked the validity of the diffusive scaling up to $t=5 \times 10^{4}$, as shown in Fig. 5 for $\rho=3 k / 5$ and $d=1$. (As long as $t \ll L^{2}=10^{6}$, finite size effects can be safely ignored.) We calculated $\frac{\left\langle\mathbf{r}^{2}\right\rangle}{2 d \cdot t}$ by using data in the $0 \leq t \leq 5 \times 10^{4}$ time window. The results are shown in Figs. 6 and 7.

As a reference point, it is useful to have a mean-field prediction. To derive the mean-field prediction for the self-diffusion coefficient of the GEP with an arbitrary maximal occupancy $k$ we recall that a site is occupied by $k$ particles with probability $P_{k}$, so it can be a destination site with probability $1-P_{k}$. Therefore for a tagged particle, the hopping rate to each neighboring site appears to be $1-P_{k}(\rho) \equiv \Lambda_{k}(\rho)$, which tells us that the self-diffusion coefficient is

$$
\mathcal{D}_{k}^{\mathrm{MF}}=\Lambda_{k}=\frac{Z_{k-1}(\lambda)}{Z_{k}(\lambda)}=\frac{\rho}{\lambda} .
$$

In particular, $\mathcal{D}_{1}^{\mathrm{MF}}=1-\rho$ for the $\mathrm{SEP}$, while for $k=2$ the mean-field prediction $\mathcal{D}_{2}^{\mathrm{MF}}=\Lambda_{2}(\rho)$ is given by the explicit formula $(26)$. For non-interacting random walks Eq. (52) yields $\mathcal{D}_{\infty}^{\mathrm{MF}}=1$, and this is the only case when the prediction is exact. In all other cases $(1 \leq k<\infty)$, the prediction of Eq. (52) is not exact.

To appreciate the mean-field nature of the prediction (52) we first note that for every site, the probability that any neighboring site contains less than $k$ particles is indeed $1-P_{k}$, and these probabilities are uncorrelated. So if we pick a particle and mark it with a tag, it appears that this particle is indeed diffusing with the coefficient equal to $1-P_{k}$. But we must keep the identity of the tagged particle. This already causes the problemimmediately after the tagged particle has undergone the first jump, the site from which it jumped will be surely

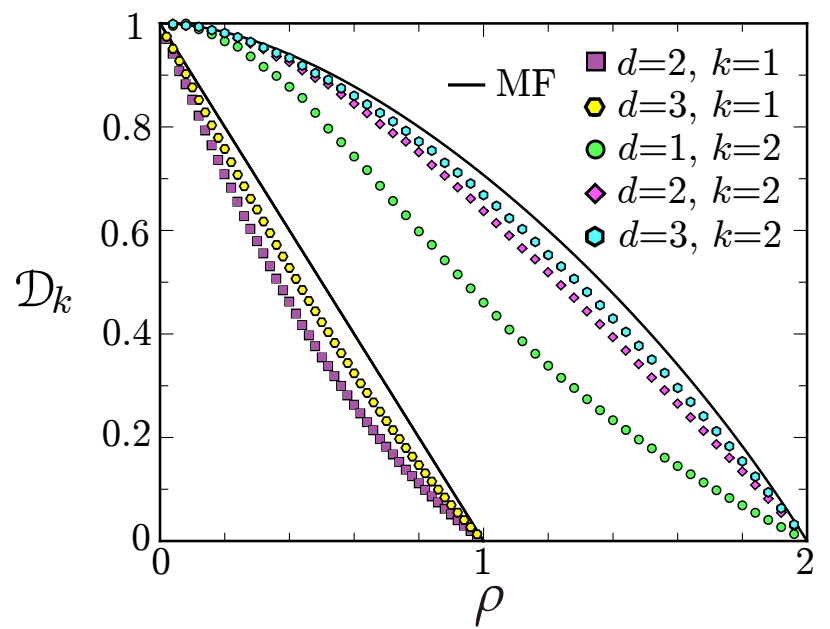

FIG. 7: Coefficient of self-diffusion vs density for the SEP in two and three dimensions, and for the GEP with $k=2$ in dimensions $d=1,2,3$. Dots represent simulation results. Solid lines are the mean-field predictions: $\mathcal{D}_{1}^{\mathrm{MF}}=1-\rho$ for the SEP, while for the GEP with $k=2$ the mean-field prediction $\mathcal{D}_{2}^{\mathrm{MF}}=\Lambda_{2}(\rho)$ acquires the explicit form 26 .

occupied by less than $k$ particles. In the $d \rightarrow \infty$ limit this is irrelevant, but for any finite dimension the derivation of Eq. (52) involves an uncontrolled approximation. We thus realize that Eq. (52) only provides a mean-field approximation. To summarize, the prediction (52) satisfies the following properties:

1. It agrees with the limiting behaviors (51).

2. It appears to be an upper bound for all $d \geq 1$.

3. It becomes exact in the $d \rightarrow \infty$ limit. This justifies calling 52 a mean-field prediction.

4. It is also exact for non-interacting random walks $(k=\infty)$.

The validity of the first property easily follows from Eq. (52), and it is also seen (in the one-dimensional case) from Fig. 6. The second property seems very plausible, but has not been proved; it is supported by simulation results; see Figs. 6 and 7 . The validity of the third and fourth properties is obvious.

Figures 6 and 7 indicate that the disagreement between the actual behaviors and the mean-field predictions is most pronounced in one dimension, so the mean-field estimate (52) provides a good approximation in two and three dimensions.

Assuming that the mean-field estimate 52 provides qualitatively correct small and large $\rho$ behaviors also in finite dimensions, we anticipate that

$$
\lim _{\rho \downarrow 0} \frac{1-\mathcal{D}_{k}(\rho, d)}{\rho^{k}}=A_{k}(d), \lim _{\rho \uparrow k} \frac{\mathcal{D}_{k}(\rho, d)}{k-\rho}=B_{k}(d) .
$$


Little is known about these amplitudes. For $d=\infty$,

$$
A_{k}(\infty)=\frac{1}{k !}, \quad B_{k}(\infty)=1,
$$

because the mean-field prediction becomes exact when $d=\infty$. Since the mean-field estimate $(52)$ apparently provides an upper bound, we expect that $A_{k}(d)>\frac{1}{k !}$ and $B_{k}(d)<1$.

\section{SUMMARY}

We investigated generalized exclusion processes with symmetric nearest-neighbor hopping parametrized by an integer $k$, the maximal occupancy. Specifically, we studied a class of such processes interpolating between the symmetric exclusion process $(k=1)$ and non-interacting random walkers $(k=\infty)$. For these lattice gases the hydrodynamic behavior is governed by a diffusion equation. We computed the diffusion coefficient $D_{k}$ and showed that for every $k$, it does not depend on the spatial dimension, but it does depend on $k$. We showed that, apart from the extreme cases of $k=1$ and $k=\infty$, the diffusion coefficient depends on the density.

We studied numerically the self-diffusion phenomenon for the GEPs in one, two, and three dimensions. An interesting challenge is to compute the self-diffusion coefficient for the GEPs with $k \geq 2$ in one dimension. In two and higher dimensions this problem seems intractable, even for the SEP in two dimensions the coefficient of selfdiffusion is unknown; it has only been established that $\mathcal{D}_{1}(\rho, d)$ is a smooth function of the density [47. In the one-dimensional setting, the tagged particle in the case of the SEP undergoes an anomalously slow sub-diffusive behavior which is well understood, and even large deviations of the displacement of the tagged particle have been probed (see e.g. [54, 55] and references therein). There- fore there is a hope that the self-diffusion phenomenon in the one-dimensional GEP is also analytically tractable.

In addition to the diffusion coefficient, a second transport coefficient, the mobility (or conductance) $\sigma(\rho)$, plays an important role in the macroscopic fluctuation theory [17. The knowledge of $\sigma(\rho)$ is required if one wants to understand fluctuations around the (deterministic) hydrodynamic behaviors, including large deviations. We leave the determination of the mobility $\sigma(\rho)$ for future studies of fluctuations and large deviations in the GEPs.

In this article we considered only the GEP with symmetric hopping. One would like to understand the asymmetric version of the GEP. The problem is that in the driven case the structure of the steady states is unknown: it is not a product measure anymore, even on a ring [22]. One possibility is to modify the rules of the GEP to make the structure of the steady states more accessible. An interesting variant is to employ a drop-push dynamics when a hopping attempt to a fully occupied neighboring site is not rejected, but instead the particle proceeds in the same direction and lands at the closest site which is not fully occupied [24, 25]; a similar process was suggested, and studied for $k=1$, in the context of self-organized criticality [45]. For these GEPs with drop-push dynamics the structure of the steady states is known in the general case of asymmetric hopping [24, 25]. The symmetric version of the GEPs with drop-push dynamics also deserves further analysis, e.g., one would like to compute the diffusion coefficient for this class of lattice gases.

Acknowledgments. One of the authors (CA) thanks Chihiro Matsui for a stimulating discussion. KM and PLK are grateful to the Galileo Galilei Institute for Theoretical Physics for the hospitality and the INFN for partial support during the completion of this work. The work of P.L.K. was partly supported by grant No. 2012145 from the US-Israel Binational Science Foundation (BSF).
[1] S. Katz, J. L. Lebowitz, and H. Spohn, J. Stat. Phys. 34, 497 (1984).

[2] H. Spohn, Large Scale Dynamics of Interacting Particles (Springer-Verlag, New York, 1991).

[3] B. Schmittmann and R. K. P. Zia, Statistical Mechanics of Driven Diffusive Systems, in: Phase Transitions and Critical Phenomena, edited by C. Domb and J. L. Lebowitz (Academic Press, London, 1995), Vol. 17.

[4] B. Derrida, Phys. Rep. 301, 65 (1998).

[5] T. M. Liggett, Stochastic Interacting Systems: Contact, Voter, and Exclusion Processes (Springer, New York, 1999).

[6] C. Kipnis and C. Landim, Scaling Limits of Interacting Particle Systems (Springer, New York, 1999).

[7] P. L. Krapivsky, S. Redner, and E. Ben-Naim, A Kinetic View of Statistical Physics (Cambridge University Press, Cambridge, UK, 2010).

[8] D. Chowdhury, L. Santen, and A Schadschneider, Phys.
Rep. 329, 199 (2000).

[9] T. Chou, K. Mallick, and R. K. P. Zia, Rep. Prog. Phys. 74, 116601 (2011).

[10] G. M. Schütz, Exactly Solvable Models for Many-Body Systems Far From Equilibrium, in Phase Transitions and Critical Phenomena, edited by C. Domb and J. L. Lebowitz (Academic Press, London, 2000), Vol. 19.

[11] R. A. Blythe and M. R. Evans, J. Phys. A 40, R333 (2007).

[12] B. Derrida, J. Stat. Mech. P07023 (2007).

[13] L. Bertini, A. De Sole, D. Gabrielli, G. Jona-Lasinio, and C. Landim, J. Stat. Phys. 107, 635 (2002).

[14] T. Bodineau and B. Derrida, C. R. Physique 8, 540 (2007).

[15] B. Derrida, J. Stat. Mech. P01030 (2011).

[16] G. Jona-Lasinio, J. Stat. Mech. P02004 (2014).

[17] L. Bertini, A. De Sole, D. Gabrielli, G. Jona-Lasinio, and C. Landim, arXiv:1404.6466 
[18] G. Gentile, Nuovo. Cim. 17, 493 (1940).

[19] A. Khare, Fractional Statistics and Quantum Theory (World Scientific, Singapore, 1997).

[20] C. Kipnis, C. Landim, and S. Olla, Comm. Pure Appl. Math. 47, 1475 (1994).

[21] C. Kipnis, C. Landim, and S. Olla, Ann. Inst. H. Poincaré Probab. Statist. 31, 191 (1995).

[22] T. Seppäläinen, Ann. Prob. 27, 361 (1999).

[23] G. M. Schütz and S. Sandow, Phys. Rev. E 49, 2726 (1994).

[24] M. Barma and R. Ramaswamy, in: Nonlinearity and Breakdown in Soft Matter, eds. B. K. Chakrabarti, K. K. Baradhan and A. Hansen (Springer, Berlin, 1994).

[25] G. M. Schütz, R. Ramaswamy, and M. Barma, J. Phys. A: Math. Gen. 29, 837 (1996).

[26] F. Tabatabaei and G. M. Schütz, Phys. Rev. E 74, 051108 (2006).

[27] U. Basu and P. K. Mohanty, Phys. Rev. E 82, 041117 (2010).

[28] J. Tailleur, J. Kurchan, and V. Lecomte, J. Phys. A: Math. Theor. 41, 505001 (2008).

[29] C. Matsui, arXiv 1311.7473 (2013).

[30] G. Carinci, C. Giardinà, F. Redig, and T. Sasamoto, arXiv:1407.3367 (2014).

[31] A. Ryabov, Phys. Rev. E 89, 022115 (2014).

[32] T. Becker, K. Nelissen, B. Cleuren, B. Partoens, and C. Van den Broeck, Phys. Rev. Lett. 111, 110601 (2013).

[33] T. Becker, K. Nelissen, B. Cleuren, B. Partoens, and C. Van den Broeck, arXiv:1410.3360 (2014).

[34] K. Mallick, S. Mallick, and N. Rajewsky, J. Phys. A: Math. Gen. 32, 8399 (1999).

[35] M. R. Evans, P. A. Ferrari, and K. Mallick, J. Stat. Phys. 135, 217 (2009).

[36] P. A. Ferrari and J. B. Martin, Ann. Probab. 35, 807 (2007).

[37] Y. Nagahata, Stoch. Process Appl. 116, 957 (2006).
[38] M. R. Evans and T. Hanney, J. Phys. A: Math. Gen. 38, R195 (2005).

[39] A. De Masi and P. A. Ferrari, J. Stat. Phys. 36, 81 (1984).

[40] More precisely, $D(\rho)>0$ away from a critical density where the equilibrium lattice gas undergoes a phase transition. Such a phase transition may occur in $d \geq 2$ dimensions; e.g., it occurs in exclusion processes with infinitely strong repulsion between particles in adjacent sites. However, there are no phase transitions in GEPs which we study. In these lattice gases a stronger bound, $D_{k}(\rho) \geq 1$, is actually valid for all $k$ and $\rho$.

[41] H. Spohn, J. Phys. A: Math. Gen. 16, 4275 (1983).

[42] P. Resibois and M. De Leener, Classical Kinetic Theory of Fluids (Wiley, New York, 1977).

[43] J. S. Hager, J. Krug, V. Popkov, and G. M. Schütz, Phys. Rev. E 63, 056110 (2001).

[44] P. L. Krapivsky, J. Stat. Mech. P06012 (2013).

[45] J. M. Carlson, J. T. Chayes, E. R. Grannan, and G. H. Swindle, Phys. Rev. Lett. 65, 2547 (1990).

[46] U. Bhat and P. L. Krapivsky, Phys. Rev. E 90, 012133 (2014).

[47] C. Landim, S. Olla, and S. R. S. Varadhan, Commun. Math. Phys. 224, 307 (2001).

[48] T. E. Harris, J. Appl. Prob. 2, 323 (1965).

[49] D. G. Levitt, Phys. Rev. A 8, 3050 (1973).

[50] P. M. Richards, Phys. Rev. B 16, 1393 (1977).

[51] S. Alexander and P. Pincus, Phys. Rev. B 18, 2011 (1978).

[52] R. Arratia, Ann. Probab. 11, 362 (1983).

[53] C. Kipnis and S. R. S. Varadhan, Commun. Math. Phys. 104, 1 (1986).

[54] S. Sethuraman and S. R. S. Varadhan, Ann. Probab. 41, 1461 (2013).

[55] P. L. Krapivsky, K. Mallick, and T. Sadhu, Phys. Rev. Lett. 113, 078101 (2014). 